\title{
Pronominalization and discourse coherence, discourse structure and pronoun interpretation
}

\author{
PETER C. GORDON and KIMBERLY A. SCEARCE \\ University of North Carolina, Chapel Hill, North Carolina
}

\begin{abstract}
Two self-paced reading-time experiments are reported that examine the time course of pronoun interpretation processes based on local discourse structure and on world knowledge. The characterization of local discourse structure is based on recent work on centering, which provides a specific formulation of how the ways in which sentences make reference to common entities determines the coherence of discourse segments and how discourse structure influences interpretation of ambiguous pronouns. The results of the first experiment show that readers generate a default interpretation of a pronoun based on features of local discourse structure, and that that default interpretation is later confirmed or overridden by knowledge-based processes. The results of the second experiment show that local discourse structure continues to influence pronoun interpretation even when the semantic information that ultimately compels interpretation occurs before the pronoun. These results support the view that processes acting on local discourse structure play a powerful role in guiding language comprehension.
\end{abstract}

Pronominal reference often plays a critical role in making discourse coherent, and the assumption that discourse is well structured is sometimes critical for the correct interpretation of pronouns. This mutual dependence reflects the fact that the structure of coherent discourse causes it to center on a particular entity: Referring to that entity with a reduced linguistic form such as a pronoun reinforces the entity's centrality; conversely, assuming the entity's centrality allows a potentially ambiguous pronoun to be interpreted on the basis of discourse structure. However, this is not the whole story; in the end, a pronoun will be interpreted in the way that is semantically most plausible given the meanings conveyed by a discourse. This raises the question of the relationship between processes of pronoun interpretation based on discourse structure and on the world knowledge that forms the basis for evaluating semantic plausibility. Here, we investigate the thesis that readily accessible features of discourse structure are used to generate a default interpretation of a pronoun independently of world knowledge, and that slower inference processes, based on world knowledge, later confirm or override that interpretation. Below, we review evidence about how patterns of coreference contribute to local discourse coherence and structure, and about how discourse structure

The research reported in this paper was supported by a grant from the National Science Foundation (IRI-90-09018) to Harvard University, Barbara J. Grosz and P.C.G., Principal Investigators. The experiments were part of a Harvard College Honors Thesis by K. A. Scearce, who is now in the Neuroscience Department, Columbia University. They also formed part of a report at the 1992 meeting of the Psychonomic Society. Direct correspondence to P. C. Gordon, Department of Psychology, University of North Carolina at Chapel Hill, Chapel Hill, NC 27599-3270 (e-mail: pcg@gibbs.oit.unc.edu). contributes to interpretation of pronouns. Then we present two self-paced reading-time experiments that track the timecourse of structure-based and knowledge-based processes in pronoun interpretation.

\section{Local Discourse Structure}

It is widely agreed that patterns of coreference play a critical role in the local coherence of discourse (e.g., Halliday \& Hassan, 1976; Johnson-Laird, 1983; Kintsch $\&$ van Dijk, 1978). The importance of continuity of reference has been demonstrated by studies showing that discourse comprehension, as measured by reading time and recall, is facilitated when successive sentences refer to the same entities (Kintsch, Kozminsky, Streby, McKoon, \& Keenan, 1975; Manelis \& Yekovich, 1976). The importance of the linguistic form of reference has been demonstrated by studies of language production showing that speakers use reduced forms (such as pronouns and ellipses) to refer to entities that are linguistically focused, while unreduced forms are used to refer to nonfocused entities (Chafe, 1976; Fletcher, 1984; MarslenWilson, Levy, \& Tyler, 1982).

The way in which these factors, linguistic form and continuity of reference, affect local discourse coherence has been captured within the theoretical framework of centering theory, which was developed within computational linguistics (Grosz, Joshi, \& Weinstein, 1983, 1986) and which has been tested and refined as a psychological theory of discourse processing (Gordon \& Chan, in press; Gordon, Grosz, \& Gilliom, 1993; Hudson-D'Zmura, 1988; Hudson-D'Zmura \& Tanenhaus, 1993; see Gordon, 1993, for a review). In the centering framework, the semantic objects referred to in utterances serve as discourse centers which are linked together to make a discourse coherent. The linguistic form used to refer to an entity is termed 
its manner of realization. An utterance in a discourse can contain two kinds of centers: a backward-looking center $(\mathrm{Cb})$ and a set of forward-looking centers (Cf).

The $\mathrm{Cb}$ is the entity in an utterance that provides a link back to the immediately preceding utterance. The construct of the $\mathrm{Cb}$ is intended to capture the role of given information (Clark \& Haviland, 1977; Haviland \& Clark, 1974; Prince, 1981) in the local coherence of discourse. According to centering theory, each utterance has only a single backward-looking center and it is crucial to discourse coherence that it be realized as a pronoun rather than as a name or full definite description. This point has been substantiated experimentally by studies (Gordon et al., 1993) showing that self-paced reading time is elevated considerably when the $\mathrm{Cb}$ is realized as a name rather than a pronoun. This elevation in reading time does not result simply from intrinsic characteristics of names as compared with pronouns (e.g., greater length and lower frequency). Rather, it is influenced by specific structural factors within and between sentences (Gordon \& Chan, in press; Gordon et al., 1993). Investigation of these structural factors has shown that the entity realized as the grammatical subject of an utterance will be its $\mathrm{Cb}$ if that is possible (Gordon et al., 1993), but that an entity realized as the direct object can take that role if the subject entity provides no possible link to the previous utterance (Gordon \& Chan, in press).

The set of forward-looking centers (Cf) in an utterance provides potential links to the subsequent utterance. It contains all the entities realized in an utterance, ranked according to their prominence within the utterance. The prominence of an entity within the $\mathrm{Cf}$ of an utterance influences the ease with which it can be the backward-looking center of the subsequent utterance. Psychologically, prominence in the Cf reflects differences in accessibility from short-term memory. In the original formulation of centering (Grosz et al., 1986), prominence was thought to be determined by such factors as surface position, grammatical role, and accent if speaking. Reading-time experiments (Gordon et al., 1993) have shown that status as grammatical subject and surface-initial position in a sentence both contribute to an entity's being highly ranked in the $\mathrm{Cf}$. These results are consistent with findings (Chang, 1980; Gernsbacher $\&$ Hargreaves, 1988) showing that the initial entity of a sentence, independent of grammatical role, is highly accessible from memory, as measured by probe reaction time tasks. The ranking of entities in the $\mathrm{Cf}$ provides a way for the $\mathrm{Cb}$ to shift smoothly from one entity to another while still maintaining coherent links among successive utterances. This ranking is also thought to provide default values for interpreting pronouns based on discourse structure. In particular, the first pronoun in an utterance will be interpreted as referring to the highest ranked member of the $\mathrm{Cf}$ of the previous utterance $\left(\mathrm{Cf}_{1}\right)$.

\section{Pronoun Interpretation}

The occurrence of a pronoun presents a reader or listener with the task of finding its semantic referent. There are a number of possible relations between a pronoun and the antecedent expression which is the central cue for determining its semantic referent. The pronoun and its antecedent can occur in the same sentence or in different sentences within the same local discourse segment. In addition, they can occur in different discourse segments, in which case interpretation of the pronoun involves reinstatement of its referent in working memory (Kintsch \& van Dijk, 1978; O'Brien, 1987). Here, we will focus on intersentential coreference within a local discourse segment, and on intrasentential coreference where the pronoun and the antecedent expression occur in different clauses connected by the relatively neutral coordinator "and." We will consider evidence from online tasks (e.g., reading time and probe tasks) and from off-line tasks (e.g., pronoun identification and fragment completion).

Logically, it would seem that the task of interpreting a pronoun would be easier when the linguistic characteristics of a pronoun itself (e.g., gender and number) match a single candidate referent rather than multiple candidate referents, or when intrasentential constraints on coreference result in a single candidate referent. In fact, empirical studies have shown that syntactically unambiguous pronouns are sometimes understood nore readily than syntactically ambiguous pronouns (Ehrlich, 1980; Fredericksen, 1981; Garnham \& Oakhill, 1985; Matthews \& Chodorow, 1988). Determination of the referent of syntactically ambiguous pronouns must rely on the constraints offered by discourse structure and by an assessment of which interpretation of a pronoun is most plausible semantically given the message being conveyed.

Previous empirical research has addressed three facets of discourse structure that potentially influence the ease of interpreting pronouns: recency, parallel function, and subject-assignment bias. According to a recency strategy, pronoun interpretation involves searching backwards through the text to find the closest realization of a potential referent (Clark \& Sengul, 1979). This strategy is supported by studies showing that sentences containing pronouns are understood more readily when prior realizations of the pronoun are near by (Clark \& Sengul, 1979; Ehrlich \& Rayner, 1983). However, other studies have shown that some kinds of primacy may be more important than recency (Cowan, 1980; Matthews \& Chodorow, 1988), and other studies have shown that distance in the text is often confounded with shifts of topic or focus (Clifton \& Ferreira, 1987a; Lesgold, Roth, $\&$ Curtis, 1979; see Garnham, 1987, for a discussion). This makes studies of distance effects difficult to interpret.

According to the parallel-function strategy, a pronoun will be interpreted as coreferential with the expression in the previous clause or sentence that has the same grammatical role (Sheldon, 1974). This can be seen as part of a general tendency in language for structures "to be interpreted in a parallel fashion unless there is external pressure for non-parallel interpretations" (Kuno, 1987, p. 7). Grober, Beardsley, and Caramazza (1978) and Ehrlich (1980) have shown that parallel function guides 
readers' interpretation of pronouns in subject position in a subordinate clause connected by "and." Fredericksen (1981) has shown that sentences with an ambiguous pronoun as grammatical subject are read more quickly when their referent was previously in subject position rather than some other position. Thus, there is some empirical support for the parallel-function strategy in pronoun interpretation.

However, these results can also be explained by the subject-assignment heuristic. This strategy (e.g., Crawley, Stevenson, \& Kleinman, 1990; Hobbs, 1976) states that an ambiguous pronoun will preferentially be interpreted as coreferential with the subject of the preceding utterance. When the pronoun in question is a grammatical subject, this strategy yields identical predictions with parallel function, and the primary experimental tests of the parallel-function strategy have looked at just such cases (Crawley et al., 1990). In an attempt to determine the relative power of subject assignment and parallel function in intrasentential reference, Crawley et al. examined readers' identifications of the referents of pronouns in object position, when either the subject or object of the immediately preceding conjoined clause could plausibly be its referent. They found a statistically significant, but not overwhelming, tendency to interpret the pronoun as coreferential with the subject. This is consistent with subject assignment rather than with parallel function.

Centering theory subsumes most instances of subjectassignment bias, and accordingly most documented instances of the parallel function heuristic. It does so because subject status and surface-initial position both contribute independently to ranking in the set of forwardlooking centers (Gordon et al., 1993). The independent contribution of these factors can be studied using sentences in which the surface-initial entity is not the subject (Gernsbacher \& Hargreaves, 1988; Gordon et al., 1993). However, the canonical constituent order of English sentences places the subject noun phrase (NP) in surface-initial position. Thus, both factors frequently combine to make the referent of the subject NP the most prominent entity in the sentence $\left(\mathrm{Cf}_{1}\right)$. Accordingly, centering theory is supported by the evidence suggesting a subject-assignment bias for pronouns.

The above evidence supporting a role for discourse structure in pronoun interpretation must be considered in light of arguments that language comprehension is highly interactive, and that knowledge-based processes play a very early role in language processing (JohnsonLaird, 1983; Marslen-Wilson \& Tyler, 1980; Tyler \& Marslen-Wilson, 1977, 1982). In an application of these principles to pronoun interpretation, Anderson, Garrod, and Sanford (1983) showed that world knowledge about the activities of certain character types constrained the interpretation of pronouns. Such findings and arguments have been used to challenge the idea that the basic linguistic processes are modular (Fodor, 1983), that is, that they make use of only a restricted class of linguistic information and proceed independently of knowledge- based cues about the likely structure of linguistic input. According to this view, the output of linguistic processing mechanisms and more general knowledge-based inference mechanisms are integrated only relatively late in processing. This position has led to a number of very closely argued debates about what kinds of information are used in comprehending linguistic input, and when they are used (e.g., Cowart \& Cairns, 1987; Rayner, Carlson, \& Frazier, 1983; Tyler \& Marslen-Wilson, 1977; see Clifton \& Ferreira, 1987b, and Garrett, 1990, for reviews). In the present paper, we extend this investigation beyond the realm of syntax, where it has principally been conducted so far, to the realm of local discourse structure. We hope to show that structural features of discourse contribute to pronoun interpretation independently of knowledge-based processes.

\section{EXPERIMENT 1}

The primary goal of this experiment was to provide a clear demonstration of the hypothesized interplay of structure-based and knowledge-based processes of pronoun interpretation: discourse structure provides the basis for an initial default interpretation of a pronoun, which can then be confirmed or overridden by knowledge-based processes. We examine this interplay in passages like the following one:

(1) Bill wanted John to look over (2) some important papers.

$\begin{array}{ll}\text { (3) He had to mail him } & \text { (4) the documents by Monday. }\end{array}$ CONTINUE

(5) Unfortunately, he/Bill $\quad$ (6) never sent the papers. SHIFT

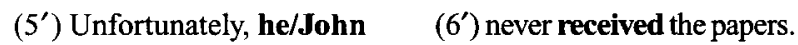
(7) As a result, the whole deal (8) fell behind schedule.

The sentences in the passage are divided in two because they were presented to readers in half-sentence frames. (The frames are numbered in parentheses.) There were four alternative versions of the third sentence (Frames 5 and 6) constructed by varying two factors. The first was the manner of realization of its grammatical subject (pronoun vs. name). The second was whether the third sentence continued or shifted the subject of the previous two sentences. This was determined semantically by the predication in the sixth frame of the passage. Frames 5 and 6 illustrate the continue version, and Frames $5^{\prime}$ and $6^{\prime}$ illustrate the shift version.

The structure of these passages maximizes the chances of the proposed interplay between structure-based and knowledge-based processes, because a large number of structural factors (primacy, subject-assignment, and parallelism-though not recency) all conspire to suggest that the pronoun at the beginning of the critical third sentence will be interpreted as coreferential with the subject of the preceding sentence. Within centering theory, all of these characterizations would be thought to reflect the ranking of the elements of the set of forward-looking centers in Sentence 2. The fact that all of these heuristics agree 
in this case is a strong indication that there is a structural bias influencing pronoun interpretation in the third sentence. In addition, the semantic information that ultimately compels the interpretation of the pronoun occurs after the referring expression. Thus, there is a period of time during which the only basis for pronoun interpretation is structural. The late positioning of the disambiguating information means that readers' interpretation of the pronoun in the shift condition may be garden-pathed by structural cues, and that this will be evident in their reading times of the second frame of that sentence.

A second goal of this experiment is to look at the differing effects of names and pronouns on discourse coherence in the continue and shift conditions. Gordon et al. (1993) have shown that there is a repeated-name penalty when the entity occurring as the grammatical subject of an utterance is realized as a name rather than a pronoun. This is interpreted within centering theory as indicating that realization with a nonreduced form such as a name prevents that entity from providing a link to the previous utterance as the backward-looking center. The current experiment goes beyond those reported in Gordon et al. (1993) by seeing whether the repeated-name penalty occurs with syntactically ambiguous pronouns in passages in which the identity of the subject sometimes shifts. One might expect that unambiguous names might have an advantage over ambiguous pronouns in such cases. In addition, this experiment allows us to see whether the repeated-name penalty occurs in a circumstance where we can simultaneously get evidence that readers in fact interpret the pronouns in the passage.

\section{Method}

Subjects. Sixteen native speakers of English attending classes at Harvard University served as subjects. They were recruited with posted notices and were paid for their participation in a single session lasting around $40 \mathrm{~min}$.

Stimulus materials. A set of 36 four-sentence passages was constructed for the experiment, with four versions of each as shown above. The four versions reflected the combination of the continue/shift and manner of realization manipulations. The majority of the passages were created specifically for this experiment, but a few included sentences adapted from Gernsbacher (1989). The first sentence of each passage introduced two characters by name, with one of the characters being the subject of the sentence. The second sentence included pronominal realizations of both characters and had the same subject as the first sentence. In the third sentence, only one of the characters was realized, and it occurred in subject position. A single word in the second half of the third sentence was varied so as to provide a semantic basis for compelling the interpretation of the subject of the third sentence. The fourth sentence did not mention either of the two characters. It was included so that the critical sentence would not be the last one in the passage. Both characters had names that were stereotypically of the same gender. Half the passages included male characters and half female characters. A true/false question was written for each passage, in order to ensure that subjects read the passages. The questions were constructed so that failure to recall the name of the character referred to by a pronoun would not result in an incorrect answer.

An off-line pilot study was conducted on the pronoun versions of the experimental passages in order to make certain that the semantic changes in the third sentence would lead to the desired pro- noun interpretation. Subjects indicated the referents of all pronouns in a passage after reading through it completely. Passages were presented in a random order with different versions of a given passage occurring in different halves of the test. Passages were tested, revised, and then retested until $90 \%$ of the subjects identified the pronouns in the expected way. Two groups of 10 subjects participated in each phase of pilot testing.

Design and Procedure. The 36 experimental passages were grouped with 108 filler passages into six blocks of 24 passages each. The first block contained only filler passages, and each of the subsequent blocks contained 6 experimental passages. Four groupings of the experimental passages were constructed so that a given subject read each experimental passage once, and read equal numbers of passages in each of the four conditions. Across subjects, each passage occurred equally often in each condition.

Each sentence in a passage was divided into two parts. The division was done so that each part was roughly equal in length and so that the break seemed natural. In the critical third sentence of the experimental passage, the first part of the sentence always contained the critical reference and the second half of the sentence always contained the single word that compelled the interpretation of the pronoun.

At the start of each trial, the words "Press any key to begin trial" were presented in the center of the screen. During a trial, the phrases from the passages were presented in the center of the screen one at a time. To move on to the next phrase, subjects had to press the space bar. Instructions to the subjects emphasized reading at a comfortable pace. They were told: "Read each passage at a natural pace. Don't hurry, but also don't delay pressing the space bar after you have read and understood the phrase." After the subject had read the passage, the screen went blank for 1 sec. Then, the true/false comprehension question appeared in the center of the screen. The subjects indicated their responses by pressing appropriately labelled keys. Feedback was given only on trials in which the response was incorrect. At the end of each block, the subjects were told how many comprehension questions they had answered incorrectly.

\section{Results}

Figure 1 shows the mean reading time for each frame after the experimental manipulations (i.e., Sentences 3 and 4), and the reading times per frame for the first and second halves of the critical third sentence are shown in Figures 2 and 3, respectively. Analyses of variance were performed by subjects, $F_{1}$, and by passage, $F_{2}$. For the first half of the critical sentence (Frame 5), sentences

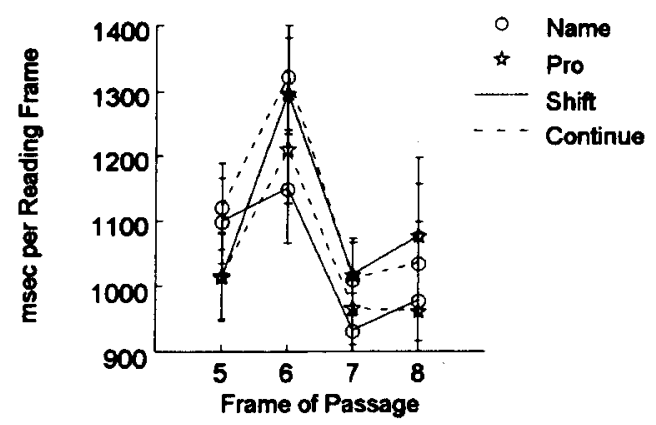

Figure 1. Experiment 1: The means and $95 \%$ confidence intervals for reading times in the third and fourth sentences (Display Frames 5-8). Frame 5 contains the critical referring expression (pronoun or name). Frame 6 contains the semantic information that disambiguates the referring expression in the preceding frame. 


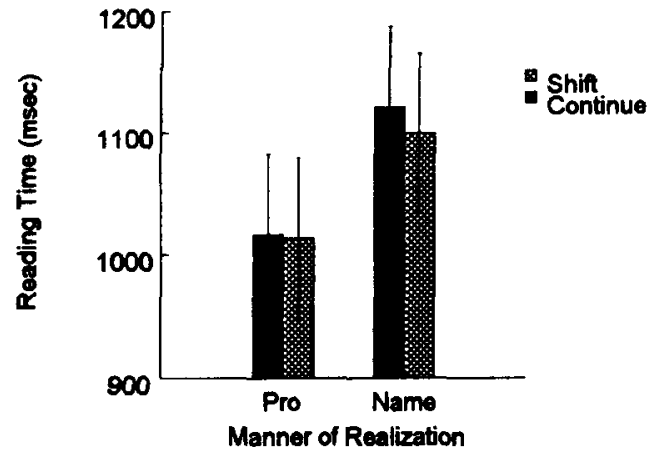

Figure 2. Experiment 1: The means and 95\% confidence intervals for reading times in the display frame containing the critical reference (the first half of the third sentence, which is Display Frame 5).

containing pronouns were read significantly faster than those containing names $\left[F_{1}(1,15)=11.7, p<.005\right.$; $\left.F_{2}(1,35)=7.8, p<.01\right]$. There was no significant effect of continue versus shift and no significant interaction between that factor and pronominalization. For the second half of the critical sentence (Frame 6), neither continue/shift nor pronominalization caused a significant main effect, but there was a significant interaction between the two variables $\left[F_{1}(1,15)=9.8, p<.01 ; F_{2}(1,35)=8.4\right.$, $p<.01]$; sentences with pronouns were read more quickly in the continue than in the shift condition, while sentences with repeated names were read more quickly in the shift than in the continue condition. ${ }^{1}$ This interaction persisted in a significant fashion in Frame $7\left[F_{1}(1,15)\right.$ $\left.=5.5, p<.05 ; F_{2}(1,35)=7.6, p<.01\right]$ and in a nonsignificant fashion in Frame $8\left[F_{1}(1,15)=2.1, p>.10\right.$; $\left.F_{2}(1,35)=6.1, p<.025\right]$. Accuracy on the comprehension questions averaged $95.6 \%$ overall, and ranged from $93.8 \%$ in the shift-name condition to $98.6 \%$ in the continue-pronoun condition. There were no significant effects of the experimental conditions on accuracy.

\section{Discussion}

The results of this experiment support the idea that readers assign a default interpretation to pronouns and then

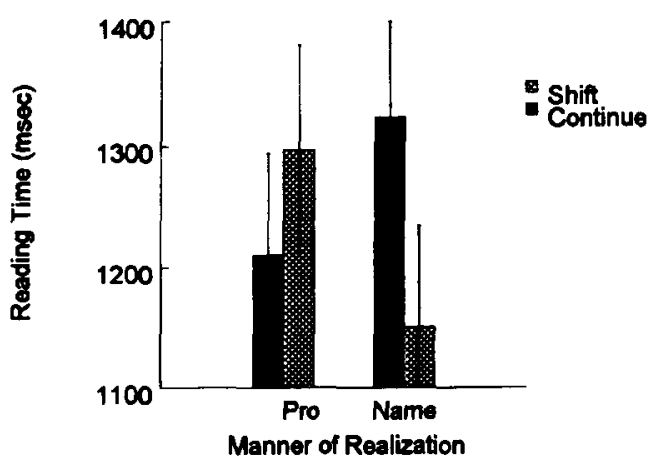

Figure 3. Experiment 1: The means and $95 \%$ confidence intervals for reading times in the display frame containing the semantically disambiguating information (the second half of the third sentence, which is Display Frame 6). confirm or override that interpretation using knowledgebased processes. This support derives from the pattern of reading times for the text containing (and following) the semantic information that disambiguates the pronoun in the first half of the critical sentence (Frame 5). The reading times for the subsequent text (Frames 6-8) are faster when the semantic information in the second half of the critical sentence confirms that the pronoun is coreferential with the subject of the first two sentences (the continue condition) as compared with when that information indicates that the pronoun is coreferential with the other character mentioned in the passage (the shift condition). No such elevation in reading times is found in the shift condition when a name is used rather than a pronoun; the use of a name provides sufficient information to identify its referent, and in that case the semantic information in Frame 6 does not force reinterpretation of an earlier referring expression. Thus, the pattern across conditions is consistent with structural garden-pathing in the interpretation of pronouns.

One of the component conclusions in our above interpretation is that readers actually do identify the referent of the pronoun in the critical sentence. This conclusion would not merit special comment if it weren't for a recent paper by Greene, McKoon, and Ratcliff (1992). They argue that under ordinary reading circumstances readers do not automatically determine the referents of pronouns. Their position is based on an exhaustive series of probeword experiments which showed that the occurrence of a pronoun did not produce any facilitation in the speed of judging that a probe word matching its referent had occurred previously in the text. On the basis of systematic empirical study, Greene et al. (1992) attribute the difference between their findings and those of earlier studies (Chang, 1980; Corbett \& Chang, 1983; Gernsbacher, 1989) to the use by previous researchers of unnaturally slow reading rates, slow responses to probes, and comprehension questions that focused attention on the identity of the pronouns. According to Greene et al. (1992), the comprehension questions in those cases provided motivation to interpret the pronouns, and slow reading rates and response times provided the strategic opportunity for that unusual goal to be pursued. When Greene et al. used presentation rates that were closer to those observed in studies of natural reading (Just \& Carpenter, 1980; Rayner, 1978), and when they did not use comprehension questions that promoted attention to pronoun identity, they found that pronouns produced no facilitation for their referents, as measured by the probe task. They interpreted this finding as indicating that in their passages both the referent and nonreferent were very salient, and therefore provided equally good matches to the pronoun (even though the pronoun matched its referent, but not its nonreferent, in gender). Given equally good matches to two discourse entities, they argued that automatic processes would not yield a unique referent for the pronoun.

The present experiment seems to satisfy Greene et al.'s (1992) criteria for studying automatic processes in read- 
ing. First, the reading times for the third and fourth sentences averaged $213 \mathrm{msec}$ per word. This is faster than the 250-msec-per-word presentation rate that Greene et al. used. Of course, the present methodology allowed readers to distribute their reading time across the words in a half sentence as they wished, while the controlled presentation rate of Greene et al. (1992) may have disrupted normal reading processes and resulted in less time being available for effective reading. Be that as it may, the mean time per word that we observed is squarely in the range ( 200 to $250 \mathrm{msec}$ ) that Greene et al. cite for normal reading rates per word. Second, the comprehension questions in the current experiment did not require pronoun interpretation, and therefore were not a source of special motivation.

With regard to stimulus materials, there are similarities and differences between ours and those of Greene et al. (1992). In our experiment, the critical pronoun was the subject of a sentence and was coreferential with the subjects of the two preceding sentences. In the relevant experiments of Greene et al., the critical pronoun was the subject of a subordinate (embedded) clause and was coreferential with the subject of the main (matrix) clause. The difference between our results and those of Greene et al. could be due to some difference between intersentential and intrasentential coreference. Or, perhaps greater structural bias was created by the referent's being realized twice in subject position in the current experiment as opposed to once in Greene et al.'s experiments. The latter possibility would fit the idea of differential discourse prominence that Greene et al. present as a major mechanism for allowing a pronoun to match its intended referent better than an unintended referent. However, the idea that the current experiment allowed matches between the pronoun and its default referent that were better than those that occurred in the Greene et al. experiments is countered by the fact that pronouns of ambiguous gender were used in the current experiment whereas unambiguous pronouns were used in Greene et al.

To our minds, the most likely basis for the difference between the studies is the methodology used for measuring pronoun interpretation; Greene et al. (1992) used a probe-word technique, while we used garden-pathing as evidenced by self-paced reading times. Neither technique provides a direct window on the result of pronoun interpretation. The probe task indicates how a word's accessibility in memory is influenced by the occurrence of a pronoun, and garden-pathing reflects the consequences of integrating the interpretation of a pronoun with other meanings in the discourse. Greene et al. considered and rejected the idea that the probe task fails to detect interpretation of the pronoun, citing studies that demonstrate its sensitivity. However, garden-pathing may be more sensitive than the probe task in this case because it involves disruption of a fluent process, while the probe task looks for facilitation in the accessibility of an already very accessible entity. ${ }^{2}$

The structural effect on pronoun interpretation that we observed in this experiment is consistent with cen- tering theory (Gordon et al., 1993; Grosz et al., 1986), but it is also consistent with the parallel function (Grober et al., 1978; Sheldon, 1974) and with subject-assignment strategies (Crawley et al., 1990; Hobbs, 1976) for pronoun interpretation. However, centering theory provides a better account of the overall pattern of results concerning the processing of referring expressions than do those heuristics, because it also accounts for the effects observed for the reading of repeated names.

The second goal of the current experiment was to see whether repeated names disrupted discourse coherence (Gordon et al., 1993) in an experiment where we had evidence, through garden-pathing, that pronouns were in fact interpreted. The results showed that in the continue condition, sentences with repeated names were read significantly more slowly than those with pronouns. This eliminates a criticism, that might have been formulated on the basis of the Greene et al. (1992) results, that Gordon et al. (1993) observed the repeated-name penalty because nominal anaphors are interpreted while pronominal ones are not, and that this extra process of interpretation produced the elevated reading times that Gordon et al. (1993) had characterized as a disruption of coherence. In any case, while such a criticism would have had to contend with the finding (Gordon et al., 1993) that the repeated-name penalty was influenced by discourse structure, the current demonstration of the repeated-name penalty in conjunction with evidence of pronoun interpretation makes the point moot. The present experiment further shows that the disruption of discourse coherence caused by a repeated name is powerful enough to be exhibited even when readers are being frequently tricked about the referents of pronouns, which might cause them to read pronouns more cautiously than names.

The current experiment adds to previous evidence that the repeated-name penalty is not due to inherent differences in the speeds of reading pronouns and names. In particular, in the continue condition the repeated-name penalty persisted well beyond the reading of the actual name (located in the first half of the sentence), to later parts of the sentence and to the first half of the subsequent sentence. This is consistent with a general disruption of comprehension processes rather than a localized disruption based on such characteristics as word length or frequency. Previously, we have found that the magnitude of the repeated-name penalty depends on the syntactic role of the referring expression within a sentence, and on its serial position and syntactic role within the preceding sentence. The observation of a repeated-name penalty depends on the particular configuration of these structural factors. If they are not appropriately configured, then no repeated-name penalty is observed (Gordon et al., 1993) or names may actually be read more quickly than pronouns (Gordon \& Chan, in press). These results indicate that the repeated-name penalty reflects characteristics of discourse structure rather than inherent characteristics of names and pronouns.

Gordon et al. (1993) interpret the repeated-name penalty within the framework of centering theory (Grosz 
et al., 1986) as indicating that a repeated name prevents the entity realized as the grammatical subject from providing a link to the previous utterance (i.e., from being its backward-looking center). Gordon et al. (1993) argue that the finding of the repeated-name penalty limits the importance of Gernsbacher's (1989) explicitness principle, which states that nominal anaphors are more effective than pronominal anaphors in enhancing the memory representations of their referents. Similarly, the repeatedname penalty suggests limitations on the generality of a claim by Greene et al. (1992) that the referents of nominal anaphors are automatically determined, while the referents of pronominal anaphors are not. Greene et al. base this claim on the finding that nominal anaphors produced facilitation for their referents in a probe task which, under the same reading conditions, pronominal anaphors did not. However, the passages that Greene et al. used to test the two kinds of anaphors are quite different. In particular, those used to test nominal anaphors appear from their example (and from the description of the same passages in Dell, McKoon, \& Ratcliff, 1983) to be almost completely lacking in referential overlap across sentences. This means that local coherence among the sentences is not possible, as it was in the pronoun studies of Greene et al. (1992) and in the current study, although there may have possibly been connections between the sentences at a more global level of discourse organization (Grosz \& Sidner, 1986; Kintsch \& van Dijk, 1978). According to centering and its related approaches (Grosz et al., 1986; Grosz \& Sidner, 1986), definite descriptions (including nominal anaphors) are interpreted in relation to the global structure of a discourse, while pronouns and other reduced expressions are interpreted in relation to the local structure of discourse.

While centering has been successful in providing a good overall account of how reading times are influenced by the interaction of manner of realization (name vs. pronoun) with center transitions (continue vs. shift), it has been less successful in accounting for the direct effects of center transitions. In the original formulation of centering (Grosz et al., 1983, 1986), it was postulated that continues were easier to process than shifts. The current experiment provided no support for that prediction; the critical sentence was read at the same speed in continue-pronoun and shift-name conditions (see Footnote 1). In previous research (Gordon et al., 1993), we have also found little support for that predicted difference in processing difficulty for continues and shifts. Those findings have led us to conclude that the effect of center transitions on ease of processing needs to be reformulated to consider a domain larger than a pair of sentences, although the effect of center transitions on manner of realization can be accounted for by the original formulation.

\section{EXPERIMENT 2}

The results of the previous experiment support the idea that readers use local discourse structure to gener- ate a default interpretation of a pronoun that is either confirmed or overridden by subsequent knowledge-based processes. However, the time course of this interaction was demonstrated in a situation where structural information was the only kind available when the pronoun occurred. Therefore, it may, to some extent, reflect the distribution of information in the passages. However, several studies of syntactic parsing have looked at whether preferred parsing patterns are influenced by semantic information that is available before or during the parse in question. Ferreira and Clifton (1986; Clifton \& Ferreira, 1987b) have provided evidence that syntactic parsing is at least partially impervious to semantic influences. In contrast, Crain and Steedman (1985) concluded that structural garden paths can be blocked by appropriate contextual cues. With specific regard to the effects of discourse structure on pronoun interpretation, Crawley et al. (1990) have contended that the structural influences are biases that have an effect only when other information about pronoun identity is absent.

The present experiment examined whether the effects of discourse structure on pronoun interpretation are blocked by conflicting semantic information. It did so by examining the reading time of passages like the following:

(1) Bill wanted John to look over (2) some important papers.

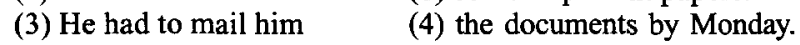

\section{CONTINUE}

(5) After sending the papers (6) he/Bill began more work. SHIFT

$\left(5^{\prime}\right)$ After receiving the papers $\left(6^{\prime}\right)$ he/John began more work. (7) When the papers are done (8) the deal will be ready to close.

The structure of the passage is the same as in the preceding experiment, except that the semantically disambiguating information was contained in a preposed phrase. If structural effects on pronoun interpretation persist in the face of preposed semantic information, then an interaction between continue versus shift and pronominalization, similar to that of Experiment 1, should be observed.

\section{Method}

Subjects. Sixteen Harvard University students who had not participated in the previous experiment served as paid subjects.

Stimuli, Procedure, and Design. The 36 experimental passages from the previous study were adapted so that the disambiguating semantic information occurred before the critical pronoun, as illustrated above. The semantic difference was generally cued by a single word change in the first half of the third sentence, but in some cases it was necessary to change two words. In addition to changes in the third sentence, adapting the passages often required changes to some other sentences so that the passage made sense. An offline pilot study was conducted in the same manner as the previous experiment, in order to ensure that the semantic differences had the intended effects on interpretation of the pronouns. Four versions of each passage were created by varying the semantic cue (continue vs. shift) and manner of realization (pronoun vs. name).

The same filler passages were used as in the previous study, and assignment of passages and subjects to experimental conditions was also done in the same manner. Subjects read the passages in a self-paced reading time experiment, with the same instructions as 


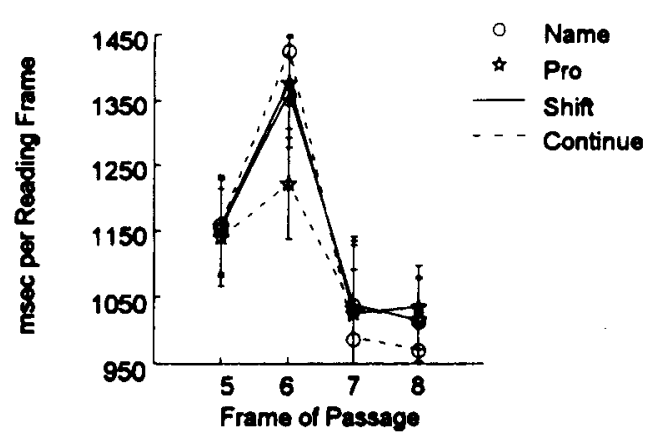

Figure 4. Experiment 2: The means and $95 \%$ confidence intervals for reading times in the third and fourth sentences (Display Frames 5-8). Frame 5 contains the semantic information that disambiguates the referring expression in the subsequent frame. Frame 6 contains the critical referring expression (pronoun or name).

before. Once again, the passages were presented a half-sentence at a time with the breaks occurring at subjectively natural boundaries. The break in the third sentence of the experimental passages always occurred between the semantic cue and the critical reference.

\section{Results}

Figure 4 shows the mean reading times for the display frames after the experimental manipulations. In the first half of the critical sentence (Frame 5), where the semantically disambiguating cue occurred, there were no significant main effects or interactions. The second half of the critical sentence (Frame 6) contained the critical referring expression, and its mean reading time as a function of pronominalization and shift versus continue is shown in Figure 5. Although the main effects of pronominalization and continue/shift were not significant, the interaction between the two factors was $\left[F_{1}(1,15)=\right.$ $\left.6.6, p<.025 ; F_{2}(1,35)=4.5, p<.05\right]$. A separate planned test examining results in the pronoun condition only showed that the continue condition was read more quickly than the shift condition $\left[F_{1}(1,15)=4.8, p<.05\right.$; $\left.F_{2}(1,35)=5.1, p<.05\right]$. For the name condition only, the shift condition was read slightly faster than the continue condition (by $65 \mathrm{msec}$ ), but this difference was not significant.

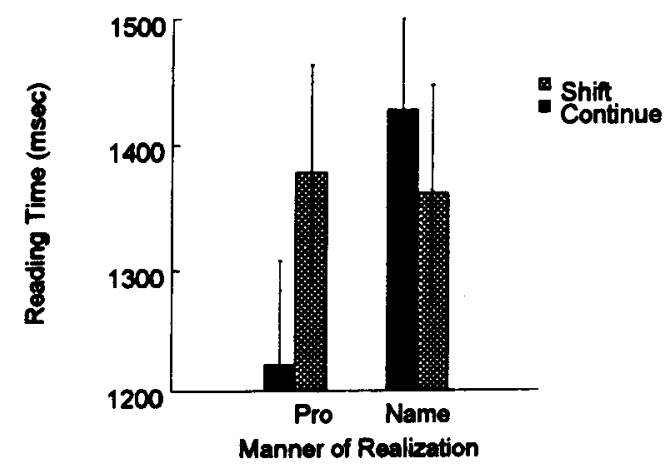

Figure 5. Experiment 2: The means and $95 \%$ confidence intervals for reading times in the display frame containing the critical reference (the second half of the third sentence, which is Display Frame 6).
There were no significant effects for either half of the final sentence (Frames 7 and 8). Accuracy on the comprehension questions averaged $94.3 \%$ overall, and ranged from 91.7 in the continue-name condition to 97.2 in the shift-name condition. These differences were not significant.

\section{Discussion}

The important results of the experiment were that there was an interaction between pronominalization and continue/shift, and that phrases containing pronouns were read more slowly in the shift condition than in the continue condition. These results support the idea that discourse structure continues to influence pronoun interpretation even when semantically disambiguating information precedes the pronoun. While our experimental manipulations ensure that the semantically disambiguating information precedes the pronoun in the text, we cannot be certain that that information had actually registered mentally before the pronoun occurred. ${ }^{3} \mathrm{Be}$ that as it may, it is clear that the process of pronoun interpretation did not take full advantage of that information.

The pattern observed in this experiment is qualitatively similar to the effects observed in the previous experiment, where discourse structure was the only information available for interpretation of the pronoun when it occurred. However, the magnitude of the effect of discourse structure on pronoun interpretation appears to be reduced somewhat by the preposing of the semantic information. In Experiment 1 the disruptive effects of gardenpathing persisted significantly into the following sentence, while in Experiment 2 it was evident only in the reading of the phrases containing the critical referring expression. This suggests that preposing the semantic information gave the general inference mechanisms using world knowledge a head start that allowed them to mitigate the disruptive effects of garden-pathing on language comprehension. Further, Experiment 1 showed a significant effect of the continue-shift manipulation within the name condition, while in Experiment 2 this difference did not reach significance. Again, this suggests that general inference mechanisms contributed to the interpretation of the critical referring expressions and made names a less appropriate manner of realization in the shift condition than they were when there was no semantic basis for shifting the backward-looking center of the discourse. That the headstart provided by preposing the semantic information did not enable those general inference mechanisms to prevent completely the discourse effects is consistent with the view that local discourse structure is a fundamental and powerful influence on language processing (Gordon et al., 1993; Grosz et al., 1986).

\section{GENERAL DISCUSSION}

The goal of this research was to examine the relationship between language comprehension processes based on local discourse structure and on world knowledge. 
The results support the view that local discourse structure plays an important role in providing default interpretations for pronouns, a role that is not obviated by the presence of conflicting semantic context which precedes the pronouns. These findings raise several questions that can be usefully discussed in relation to the issue of the modularity of language (Fodor, 1983).

One very basic question is: if language is a module, what is its domain of operation? We believe that it is incorrect to limit that domain to the sentence, and that local discourse segments have a rich structure. This structure may not be quite so intuitively salient as the syntactic structure of sentences, but it is real, and efforts at theoretically characterizing it have shown signs of success (Gordon, 1993; Gordon et al., 1993; Grosz et al., 1986). This is not to say that exactly the same module necessarily performs syntactic and discourse processing. Rather, it is to argue that well-formed language has a regular structure at the level of local discourse. Such a well-formed structure built on a constrained class of representations is what makes modular processing valuable, and it appears that readers systematically take advantage of that structure in the case of local discourse structure.

A second question has to do with the kinds of data that can be interpreted as providing evidence of modularity. Proponents of modularity (e.g., Fodor, 1983; Fodor, Garrett, \& Swinney, 1992) argue that the essential question is whether a process is informationally encapsulated, meaning that it is unaffected by potentially relevant information that is outside of its domain of operation. Of course, modularists believe that the output of modular mechanisms is eventually combined with the output of other mechanisms at higher levels of processing. This leads to the requirement that claims against modularity must provide evidence of interactive processing very early in the time course of comprehension. This requirement is one reason why strong claims of modularity or interaction are methodologically sticky. The results of the present experiments are mixed with regard to informational encapsulation. Comparison of Figures 4 and 1 suggest that there is less structural garden-pathing in Experiment 2, where semantic context preceded the pronoun, than in Experiment 1, where it did not. This could be taken as evidence that structure-based pronoun interpretation processes are not completely encapsulated. Our view is that the finding of any structural garden-pathing, when the semantic information that ultimately compels pronoun interpretation is present before the pronoun, is testimony to the use of discourse structure as a basis for organizing the processing of linguistic input.

The methodological questions surrounding study of modularity in reading are further complicated by the arguments developed by Greene et al. (1992) and McKoon and Ratcliff (1992). Although we disagree with Greene et al. about the specific circumstances under which pronouns are likely to be interpreted (see the discussion of Experiment 1), we believe that these authors have made very important arguments about the effects of goals and available processing time on the kinds of inferences that are drawn during reading. Under ordinary circumstances, readers generally have a good deal of control over how quickly they read, which greatly complicates the issue of how to measure an effect early in processing. Given the findings and arguments of Greene et al. (1992) and McKoon and Ratcliff (1992), we would not be surprised if a very deliberate pace would allow readers to avoid the kind of garden-pathing we have demonstrated.

A final question has to do with motivations for considering that mental processes are modular. These range from the purely methodological belief that large problems must be broken into small pieces in order to be tackled scientifically, to strong theoretical beliefs about issues such as brain organization and nativism. Our motivation for considering the modularity of discourse processes is that language comprehension should not be considered an unconstrained problem of general inference; doing so would make it unsolvably hard. Rather, language is structured so as to invite certain inferences and to make others unlikely. This idea is strongly supported by research as diverse as Grice's (1975) philosophical analysis of conversation and McKoon and Ratcliff's (1992) experimental data in support of their minimalist account of inference during reading. The reason that features of language like syntax and local discourse structure contribute to early levels of language processing is that they are relatively simple compared to something like world knowledge. That simpler (though not really simple) information provides a framework that constrains the inferential work that must be done using world knowledge.

\section{REFERENCES}

ANDERSon, A., Garrod, S., \& SAnford, A. (1983). The accessibility of pronominal antecedents as a function of episode shifts in narrative text. Quarterly Journal of Experimental Psychology, 35A, 427-440.

ChafE, W. L. (1976). Givenness, contrastiveness, definiteness, subjects, topics, and points of view. In C. N. Li (Ed.), Subject and topic (pp. 25-26). New York: Academic Press.

Chang, F. R. (1980). Active memory processes in visual sentence comprehension: Clause effects and pronominal reference. Memory \& Cognition, 8, 58-64.

Clark, H. H., \& Haviland, S. E. (1977). Comprehension and the given-new contract. In R. Freedle (Ed.), Discourse production and comprehension (pp. 1-40). New York: Academic Press.

Clark, H. H., \& Sengul, C. J. (1979). In search of referents for noun phrases and pronouns. Memory \& Cognition, 7, 35-41.

Clifton, C., JR., \& Ferreira, F. (1987a). Discourse structure and anaphora: Some experimental results. In M. Coltheart (Ed.), Attention and performance XII (pp. 635-654). Hillsdale, NJ: Erlbaum.

Clifton, C., \& Ferreira, F. (1987b). Modularity in sentence comprehension. In J. Garfield (Ed.), Modularity in knowledge representation and natural language understanding (pp. 277-290). Cambridge, MA: MIT Press.

Corbett, A. T., \& Chang, F. R. (1983). Pronoun disambiguation: Accessing potential antecedents. Memory \& Cognition, 11, 283-294.

CowAN, J. R. (1980). The significance of parallel function in the assignment of intrasentential anaphora. In J. Kreiman \& A. E. Ojeda (Eds.), Papers from the parasession on pronouns and anaphora (pp. 110-124). Chicago: Chicago Linguistic Society.

CoWART, W., \& CaIRNS, H. (1987). Evidence for an anaphoric mechanism within syntactic processing: Some reference relations defy semantic and pragmatic constraints. Memory \& Cognition, 15, 318-331. Crain, S., \& Steedman, M. (1985). On not being led up the garden 
path: The use of context by the psychological syntax processor. In D. Dowty, L. Karttununen, \& A. Zwicky (Eds.), Natural language parsing (pp. 320-358). Cambridge: Cambridge University Press.

Crawley, R. A., Stevenson, R. J., \& Kleinman, D. (1990). The use of heuristic strategies in the interpretation of pronouns. Journal of Psycholinguistic Research, 19, 245-264.

Dell, G. S., McKoon, G., \& Ratcliff, R. (1983). The activation of antecedent information during the processing of anaphoric reference in reading. Journal of Verbal Learning \& Verbal Behavior, 22, 121-132.

EHRLICH, K. (1980). Comprehension of pronouns. Quarterly Journal of Experimental Psychology, 32A, 247-255.

EHRLICH, K., \& RAYNER, K. (1983). Pronoun assignment and semantic integration during reading: Eye movements and immediacy of processing. Journal of Verbal Learning \& Verbal Behavior, 22, 75-87.

FerReira, F., \& Clifton, C. (1986). The independence of syntactic processing. Journal of Memory \& Language, 25, 348-368.

FleTCHER, C. R. (1984). Markedness and topic continuity in discourse processing. Journal of Verbal Learning \& Verbal Behavior, 23, 487-493.

FoDOR, J. (1983). The modularity of mind. Cambridge, MA: MIT Press.

FoDOR, J., GarReTT, M. F., \& SwINNEY, D. (1992). A modular effect in parsing. Unpublished manuscript.

FREDERICKSEN, J. R. (1981). Understanding anaphora: Rules used by readers in assigning pronominal referents. Discourse Processes, $\mathbf{4}$, 323-347.

GaRnhaM, A. (1987). Understanding anaphora. In A. W. Ellis (Ed.), Progress in the psychology of language (Vol. 3, pp. 253-300). Hove, U.K.: Erlbaum.

GaRNHAM, A., \& OAKHILL, J. (1985). On-line resolution of anaphoric pronouns: Effects of inference making and verb semantics. British Journal of Psychology, 76, 385-393.

GARRETT, M. F. (1990). Sentence processing. In D. N. Osherson \& H. Lasnik (Eds.), An invitation to cognitive science: Language (pp. 133-175). Cambridge, MA: MIT Press.

GERNSBACHER, M. A. (1989). Mechanisms that improve referential access. Cognition, 32, 99-156.

Gernsbacher, M. A., \& Hargreaves, D. (1988). Accessing sentence participants: The advantage of first mention. Journal of Memory \& Language, 27, 699-717.

Gordon, P. C. (1993). Computational and psychological models of discourse. In H. Brownell \& Y. Joanette (Eds.), Narrative discourse in normal aging and neurologically impaired adults (pp. 23-46). San Diego, CA: Singular.

Gordon, P. C., \& Chan, D. (in press). Pronouns, passives and discourse coherence. Journal of Memory \& Language.

Gordon, P. C., Grosz, B. J., \& Gilliom, L. A. (1993). Pronouns, names, and the centering of attention in discourse. Cognitive Science, 17, 311-347.

Greene, S. B., McKoon, G., \& RatclifF, R. (1992). Pronoun resolution and discourse models. Journal of Experimental Psychology: Learning, Memory, \& Cognition, 18, 266-283.

Grice, H. P. (1975). Logic and conversation. In P. Cole \& J. L. Morgan (Eds.), Syntax and semantics: Vol. 3. Speech acts (pp. 41-58). New York: Academic Press.

Grober, E. H., Beardsley, W., \& Caramazza, A. (1978). Factors influencing assignment of pronoun antecedents. Cognition, 3, 227-243.

Grosz, B. J., Joshi, A. K., \& Weinstein, S. (1983). Providing a unified account of definite noun phrases in discourse. In Proceedings of the 21st Annual Meeting of the Association for Computational Linguistics (pp. 41-50). Cambridge, MA: ACL.

Grosz, B. J., Joshi, A. K., \& WeInSteIn, S. (1986). Towards a computational theory of discourse interpretation. Unpublished manuscript.

Grosz, B. J., \& SidNER, C. L. (1986). Attention, intentions, and the structure of discourse. Computational Linguistics, 12, 175-204.

Halliday, M. A., \& Hassan, R. (1976). Cohesion in English. London: Longman.

Haviland, S. E., \& ClaRK, H. H. (1974). What's new? Acquiring new information as a process in comprehension. Journal of Verbal Learning \& Verbal Behavior, 13, 512-521.

Hoвbs, J. R. (1976). Pronoun resolution (Res. Rep. No. 76-1). New York: City University of New York, Department of Computer Sciences.
HUDSON-D'ZMURA, S. B. (1988). The structure of discourse and anaphore resolution: The discourse center and the roles of nouns and pronouns. Unpublished doctoral dissertation, University of Rochester Hudson-D'Zmura, S. B., \& Tanenhaus, M. K. (1993, May). Discourse constraints on anaphoric processing. Paper presented at the Workshop on Centering Theory in Naturally-Occurring Discourse. University of Pennsylvania, Philadelphia.

Johnson-LAIRD, P. N. (1983). Mental models: Toward a cognitive science of language, inference and consciousness. Cambridge, MA: Harvard University Press.

JuST, M. A., \& CARPENTER, P. A. (1980). A theory of reading: From eye fixations to comprehension. Psychological Review, 87, 329-354.

Kintsch, W., Kozminsky, E., Streby, W. J., McKoon, F., \& Keenan, J. M. (1975). Comprehension and recall of text as a function of content variables. Journal of Verbal Learning \& Verbal Behavior, 14, 196-214.

Kintsch, W., \& van Dijk, T. A. (1978). Toward a model of text comprehension and production. Psychological Review, 85, 363-394.

Kuno, S. (1987). Functional syntax. Chicago: University of Chicago Press.

Lesgold, A. M., Roth, S. F., \& CuRTis, M. E. (1979). Foregrounding effects in discourse comprehension. Journal of Verbal Learning \& Verbal Behavior, 18, 291-308.

MANELIS, L., \& YeKoviCH, F. R. (1976). Repetition of propositional arguments in sentences. Journal of Verbal Learning \& Verbal Behavior, 15, 301-312.

Marslen-Wilson, W., Levy, W., \& Tyler, L. K. (1982). Producing interpretable discourse: The establishment and maintenance of reference. In R. Jarvella \& W. Klein (Eds.), Speech, place, and action (pp. 339-378). New York: Wiley.

Marslen-Wilson, W., \& TYler, L. K. (1980). Towards a psychological basis for a theory of anaphora. In J. Kreiman \& A. E. Ojeda (Eds.), Papers from the parasession on pronouns and anaphora (pp. 258286). Chicago: Chicago Linguistic Society.

Matthews, A., \& Chodorow, M. S. (1988). Pronoun resolution in two-clause sentences: Effects of ambiguity, antecedent location, and depth of embedding. Journal of Memory \& Language, 27, 245-260.

McKoon, G., GreENE, S. B., \& RatclifF, R. (1992, November). Discourse models, pronoun resolution, and the implicit causality of verbs. Poster presented at the meeting of the Psychonomic Society, St. Louis.

McKoon, G., \& Ratcliff, R. (1992). Inference during reading. Psychological Review, 99, 440-466.

O'BrIEN, E. J. (1987). Antecedent search processes and the structure of text. Journal of Experimental Psychology: Learning, Memory, \& Cognition, 13, 278-290.

PRINCE, E. F. (1981). Toward a taxonomy of given-new information. In P. Cole (Ed.), Radical pragmatics (pp. 223-255). New York: Academic Press.

PYLYSHYN, Z. W. (1984). Computation and cognition: Toward a foundation for cognitive science. Cambridge, MA: MIT Press.

RAYNER, K. (1978). Eye movements in reading and information processing. Psychological Bulletin, 85, 618-660.

Rayner, K., Carlson, M., \& Frazier, L. (1983). The interaction of syntax and semantics during sentence processing: Eye movements in the analysis of semantically biased sentences. Journal of Verbal Learning \& Verbal Behavior, 22, 358-374.

SHELDON, A. (1974). The role of parallel function in the acquisition of relative clauses in English. Journal of Verbal Learning \& Verbal Behavior, 13, 272-281.

Tyler, L. K., \& MARSLEN-Wilson, W. D. (1977). The on-line effects of semantic context on syntactic processing. Journal of Verbal Learning \& Verbal Behavior, 16, 683-692.

TYLER, L. K., \& MARSLEN-Wilson, W. D. (1982). The resolution of discourse anaphors: Some on-line studies. Text, 2, 263-291.

\section{NOTES}

1. It may appear anomalous that the sixth frame is read more quickly (by $60 \mathrm{msec}$ ) in the shift-name condition than in the continue-pro condition. This anomaly is reduced somewhat by the fact that the fifth frame (the first half of the sentence) is read more slowly (by $84 \mathrm{msec}$ ) 
in the shift-name condition than in the continue-pro condition. Thus, the entire sentence is read at pretty much the same speed in the shiftname and continue-pro conditions.

2. McKoon, Greene, and Ratcliff (1992) show that the probe task does provide evidence of pronoun interpretation at fast reading rates under a particular set of circumstances. This evidence is found when the pronoun occurs in the subordinate clause of a sentence conjoined with the causal connective "because" to a matrix clause that contains a verb with implicit causality. Both the particular verb type and the causal connective are required for facilitation in the probe task to occur. The current study examined intersentential reference and therefore did not include a causal connective. Furthermore, the verbs in our passages were not selected with any particular type in mind. Accordingly, it seems more appropriate to compare our results to those of Greene et al. (1992) than to those of McKoon et al. (1992).

3 . The disambiguating information in the fronted expressions resulted from how the semantics of a nonfinite verb (gerund or infini- tive) combined with the scenario described by the passage to indicate the referent of its implicit subject (PRO). The semantic information was either directly in the meaning of the verb (e.g., "sending" vs. "receiving") or was in a predicate adjective (e.g., "being late" vs. "being early"). The structure of the sentences then indicated that the PRO be coindexed with the overt pronoun that was the subject of the sentence. A study by Fodor, Garrett, and Swinney (1992) used cross-modal priming to examine when the referent of PRO is determined psychologically. The results of the experiment indicate that the referent of PRO is not determined immediately, whereas the referent of an unambiguous overt pronoun is. The study did not provide a strong basis for determining what features of a sentence trigger search for the referent of PRO.

(Manuscript received January 19, 1994; revision accepted for publication June 8, 1994.) 